Gebhardt, Michael; Gerstner, Nico; Thorenz, Carsten

Der Einfluss über- und unterströmter Wehrverschlüsse auf den Sedimenttransport durch Stauhaltungen

Verfügbar unter / Available at:

https://hdl.handle.net/20.500.11970/100679

Vorgeschlagene Zitierweise / Suggested citation:

Gebhardt, Michael; Gerstner, Nico; Thorenz, Carsten (2013): Der Einfluss über- und unterströmter Wehrverschlüsse auf den Sedimenttransport durch Stauhaltungen. In: Wasserwirtschaft J. 103 (11/2013). S. 16-22. 


\title{
Autorenfassung
}

Gebhardt, Gerstner, Thorenz: Der Einfluss über- und unterströmter Wehrverschlüsse auf den Sedimenttransport durch Stauhal tungen, 2014

Erstveröffentlichung in Wasserwirtschaft (2014), S. 16-22.

Für eine korrekte Zitierbarkeit ist die Seitennummerierung der Originalveröffentlichung für jede Seite kenntlich gemacht.

\section{Der Einfluss über- und unterströmter Wehrverschlüsse auf den Sedimenttransport durch Stauhaltungen}

\author{
Michael Gebhardta, Nico Gerstnerb und Carsten Thorenz ${ }^{c}$ \\ Kußmaulstr. 17, 76187 Karlsruhe, \\ a michael.gebhardt@baw.de \\ b nico.gerstner@baw.de \\ c carsten.thorenz@baw.de
}

Bei aktuellen Planungen von Ersatzneubauten wird immer häufiger die Frage nach der Gestaltung der Wehranlage und der Wahl der Verschlüsse gestellt, da die europäische Wasserrahmenrichtlinie neben der ungestörten Migration aquatischer Organismen auch den ungestörten Transport von Sedimenten durch Staustufen fordert. Der vorliegende Artikel soll im Allgemeinen zum Verständnis des Sedimenttransports durch Stauhaltungen beitragen sowie im Speziellen den Einfluss über- und unterströmter Wehrverschlüsse auf den Sedimenttransport quantifizieren.

\section{Hintergrund}

In dem seit 1. März 2010 geltenden Gesetz zur Neuregelung des Wasserrechts (WHG) verpflichtet sich auch die Wasser- und Schifffahrtsverwaltung des Bundes (WSV) zur Erhaltung oder Wiederherstellung der Durchgängigkeit an den Staustufen der Bundeswasserstraßen. Nach § 34 (Durchgängigkeit oberirdischer Gewässer) ist bei Errichtung, wesentlicher Änderung oder Betrieb einer Staustufe durch geeignete Einrichtungen und Betriebsweisen die Durchgängigkeit des Gewässers zu erhalten oder wiederherzustellen. Bestehende Anlagen sind dabei nachzurüsten. Die Durchgängigkeit stellt hierbei nach der europäischen Wasserrahmenrichtlinie eine „hydromorphologische Qualitätskomponente" dar, die sowohl eine ungestörte Migration aquatischer Organismen als auch den Transport von Sedimenten beinhaltet.

\section{Sedimenttransport in staugeregelten Fließgewässern}

An staugeregelten Gewässern ist das natürliche dynamische Transportgleichgewicht in den meisten Fällen gestört, da die Verringerung der Transportkapazität eine allmählich fortschreitende Verlandung vieler Stauräume zur Folge hat [1]. Die Stauregulierung unterbricht zum einen den Transport von suspendiertem Bettmaterial und von Geschiebe. Zum anderen ist die Ablagerung von feinkörnigen Schwebstoffen als die Hauptursache der Verschlammung und als Kontamination von Sedimentablagerungen verantwortlich [10]. 


\section{Autorenfassung}

Gebhardt, Gerstner, Thorenz: Der Einfluss über- und unterströmter Wehrverschlüsse auf den Sedimenttransport durch Stauhal tungen, 2014

Unter hydraulischen Gesichtspunkten treten in einer Stauhaltung bei niedrigen und mittleren Abflüssen bis zur Stauwurzel rückgestaute Strömungsverhältnisse auf. Die Stauwurzel reicht dabei sehr häufig bis zum Oberlieger. Die Transportkapazität der Strömung und die Sohlenschubspannung nimmt dadurch in Fließrichtung ab, wobei sich die gröberen Bestandteile an der Stauwurzel ablagern (Deltabildung) und die feineren Partikel weiter unterstrom absinken. Kern [5] zeigt am Beispiel des Neckars, dass Stauhaltungen zwischen frei fließenden Flüssen und den vollkommen gestauten Talsperren bzw. natürlichen Stillgewässern einzuordnen sind: Bei schwacher bis moderater Durchströmung findeteine Sedimentation statt, während bei Hochwasser abgelagerte Sedimente erodiert und ausgespült werden. Flussstauhaltungen stellen daher auch einen Zwischenspeicher für Schwebstoffe dar. Die Verlandungstendenzen in Stauhaltungen können dabei sehr unterschiedlich sein: Beispielsweise weisen im Neckar die Stauhaltungen Lauffen, Poppenweiler und Hofen die stärksten Verlandungstendenzen auf. Dies wird von Kern [5] durch die unzureichende Ausräumung der zwischenzeitlich gespeicherten Sedimente erklärt, als Ergebnis der Flussmorphologie, der räumlichen Anordnung der Staustufen und der Stauhöhen.

\section{3 Über- und unterströmte Verschlusstypen in der WSV}

An den mehr als 330 Staustufen der Bundeswasserstraßen sind heute im Prinzip alle bekannten Verschlusstypen (Bild 1) zu finden, wobei unterströmte Verschlüsse aufgrund der Anforderungen an die Feinregulierung immer mit überströmten Verschlüssen, wie z. B. Aufsatzklappen, kombiniert werden. Bei den Standardisierungsbestrebungen in der WSV zeichnet sich ab, dass in Zukunft Druck- oder Zugsegmente sowie Schlauchwehre die Verschlusstypen der Wahl sein werden. Zur Reduzierung der Verschlusshöhe werden die beweglichen Wehre wo immer möglich mit einem Wehrhöcker, der sog. Jambor-Schwelle kombiniert. Mit der entwickelten Form kann die Verschlusshöhe bei nahezu unveränderter hydraulischer Leistungsfähigkeit um etwa 25 bis $30 \%$ reduziert werden. In Laboruntersuchungen wurde auch die Sedimentdurchgängigkeit für Sieblinien bis $110 \mathrm{~mm}$ nachgewiesen [3], [4].

\section{Numerische Untersuchungen}

\subsection{Beschreibung des numerischen Modells}

Die Berechnungen wurden mit Hilfe des Open-Source-Programms OpenFOAM durchgeführt. OpenFOAM arbeitet mit der Finite-Volumen-Methode, wobei die 


\section{Autorenfassung}

Gebhardt, Gerstner, Thorenz: Der Einfluss über- und unterströmter Wehrverschlüsse auf den Sedimenttransport durch Stauhal tungen, 2014

Gebhardt, Gerstner, Thorenz: Der Einfluss über- und unterströmter Wehrverschlüsse auf den Sedimenttransport durch Stauhaltungen.

WasserWirtschaft 11 (2014), S. 16-22.

Kontrollvolumen angewandt wird [2]. Die turbulente Strömung des 2-Phasenmodells wird durch eine Grobstruktursimulation modelliert. Die groben Wirbelstrukturen der Strömung werden durch Lösung der Navier-Stokes-Gleichungen abgebildet, während kleinskalige Wirbel durch das SpalartAllmaras-Modell angenähert werden. Die Luft-Wasser-Verteilung des 2-Phasenmodells wird mit der Volume-of-Fluid-Methode bestimmt. Die Erhaltungsgleichungen werden für das Fluidgemisch gelöst.

Das numerische Modell bildet eine 130,0 m lange, 1,0 m breite und 10,0 m hohe Rinne ab, in der sich 80,0 m nach dem Einlass ein Schütz in Form einer 0,2 m dicken Tafel befindet. Die vertikale Lage des Schützes wird an die zu simulierenden Öffnungsweiten respektive Überfallhöhen angepasst (Bild 2). Da die strömungsdynamischen Effekte in x- und z-Richtung dominieren, kann die Untersuchung an einem Ausschnittsmodell durchgeführtwerden. Die Modelllänge wurde gewählt, um einen Einfluss der ober- und unterstromigen Randbedingungen auf das Abflussgeschehen am Wehrkörper auszuschließen. Dennoch konnte in einzelnen Zuständen eine Wellenbildung im Unterwasser beobachtet werden, die durch Wellenreflexionen am Auslass des Modells entstehen. Durch eine zeitliche Mittelwertbildung wurde dieser Einfluss minimiert.

Das Berechnungsgitter basiert auf einem äquidistanten Hexaedergitter mit Zellkantenlängen von 0,50 m. Innerhalb dieses Grundgitters werden Volumina definiert, in denen das Berechnungsgitter verfeinert wird. Die Stufe der Verfeinerung richtet sich dabei nach dem Gradienten der zu untersuchenden Größe, hier das Geschwindigkeitsfeld im Nahbereich des Wehres. Da im Nahbereich des Wehrkörpers die größten Geschwindigkeitsgradienten auftreten, werden die Gitterzellen ab einer Entfernung von 15,0 m von der Wehröffnung stufenweise auf eine Kantenlänge von 6,25 cm verfeinert. Der linke und rechte Modellrand sowie die Modellsohle werden als reibungsfreiangenommen. Über den Einlass werden Durchflüsse von $Q=3,5,4,0$ und 4,5 $\mathrm{m}^{3} / \mathrm{s}$ vorgegeben. Im Falle des unterströmten Schützes wird die unterwasserseitige Randbedingung mit Wassertiefen von h2 = 2,0, 3,5 und 4,0 m variiert. Für das überströmte Schütz wird der Unterwasserstand so gewählt, dass sich ein vollkommener Überfall einstellen kann. Auf eine seitliche Belüftung wurde verzichtet. Der Atmosphärenrand des Modells wird durch eine Druckrandbedingung offen gestaltet.

\subsection{Auswertungsmethodik}

Von Interesse sind insbesondere die sohlennahen Geschwindigkeiten, da diese ein Indikator für Sedimentation bzw. Erosion sind. Die Fließgeschwindigkeiten v wurden aus den Simulationsergebnissen alle $6,5 \mathrm{~cm}$ bestimmt und mit einer Referenzgeschwindigkeit $v_{\text {ref }}$ in einem vom Wehr unbeeinflussten Strömungsbereich, etwa 


\section{Autorenfassung}

Gebhardt, Gerstner, Thorenz: Der Einfluss über- und unterströmter Wehrverschlüsse auf den Sedimenttransport durch Stauhal tungen, 2014
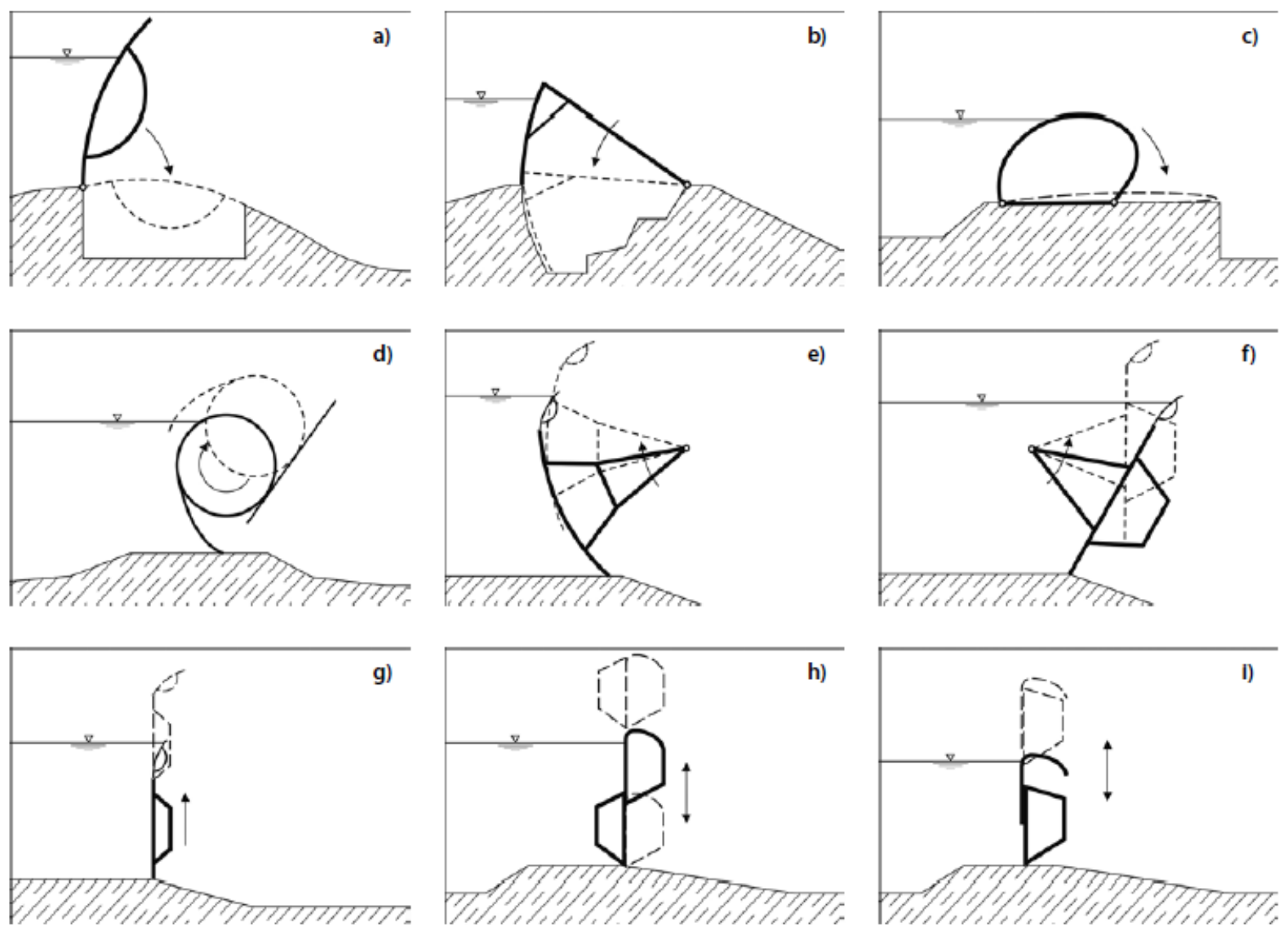

Bild 1: Verschlusstypen an Wehranlagen der WSV: a) Klappe, b) Sektor, c) Schlauch, d) Walze, e) Drucksegment mit Aufsatzklappe, f) Zugsegment mit Aufsatzklappe, g) Schütz mit Aufsatzklappe, h) Doppelschütz, i) Haken-Doppelschütz 


\section{Autorenfassung}

Gebhardt, Gerstner, Thorenz: Der Einfluss über- und unterströmter Wehrverschlüsse auf den Sedimenttransport durch Stauhal tungen, 2014

Gebhardt, Gerster, Thorenz: Der Einfluss über- und unterströmter Wehrverschlüsse auf den Sedimenttransport durch Stauhaltungen. WasserWirtschaft 11 (2014), S. 16-22.

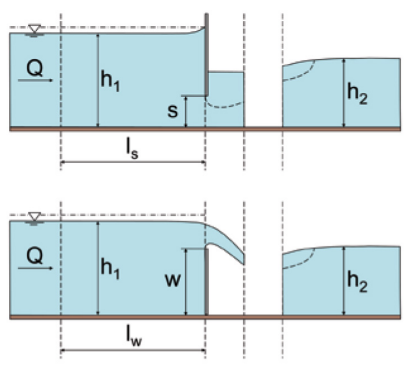

Bild 2: $\quad$ Systemskizze für ein unterströmtes (oben) und überströmtes Schütz (unten)

$60 \mathrm{~m}$ oberstrom, verglichen. Da sich die Fließgeschwindigkeit, ausgehend vom Wehr, asymptotisch der oberstromigen Referenzgeschwindigkeit annähert, wird die Einflusslänge des Wehres auf die Geschwindigkeitsverteilung über eine vorgegebene Abweichung v/vref bestimmt. Beim unterströmten Schütz werden die Abweichungen im Bereich v/Vref = 1,02 bis 1,50 vorgegeben und die entsprechende Einflusslänge mit $l_{s}$ bezeichnet. Beim überströmten Schütz liegen die Abweichungen im Bereich $v / v_{\text {ref }}=0,50$ bis 0,98 und die Einflusslänge wird mit $l_{w}$ bezeichnet.

\section{Auswertung der Simulationsergebnisse}

\subsection{Unterströmtes Schütz}

In Bild 3 sind die Isolinien der Fließgeschwindigkeiten bei einem unterströmten Schütz für drei exemplarischeZustände dargestellt. Bei konstantem Abfluss nimmt die Schützöffnungsweite s von a) nach c) zu. Am Wasserstand unmittelbar unterhalb des Schützes ist zu erkennen, dass immer rückgestauter Abfluss vorliegt. Der Oberwasserstand $\mathrm{h}_{1}$ verringert sich mit zunehmender Schützöffnungsweite und die Fließgeschwindigkeiten im Oberwasser erhöhen sich, wie beispielsweise an der Isolinie für $\mathrm{v}=1,0 \mathrm{~m} / \mathrm{s}$ gut zu erkennen ist.

Insgesamt wurden neunzehn Zustände untersucht, mit Schützöffnungsweiten zwischen 0,6 und 2,0 $m$ in Kombination mit den drei o. g. Abflüssen und Unterwasserständen. Bild 4 zeigt die daraus berechneten Abflussbeiwerte $c_{q}$ in Abhängigkeit vom dimensionslosen Ober- und Unterwasserstand $\mathrm{h}_{1} / \mathrm{s}$ bzw. $\mathrm{h}_{2} / \mathrm{s}$ nach der bekannten Gleichung:

$$
c_{q}=\frac{Q}{b \cdot s \sqrt{2 \cdot g \cdot h_{1}}}
$$




\section{Autorenfassung}

Gebhardt, Gerstner, Thorenz: Der Einfluss über- und unterströmter Wehrverschlüsse auf den Sedimenttransport durch Stauhal tungen, 2014

Dabei ist zu erkennen, dass bei allen untersuchten Zuständen ein Rückstaueinfluss vorlag, da der Abflussbeiwert niedriger ist als der theoretisch bzw. experimentell bestimmte Abflussbeiwert für ein Freispiegelschütz mit freiem Abfluss [6]. Mit zunehmendem $\mathrm{h}_{1}$ /s nähern sich beide Kurven einander an. Da die Einflusslänge $l_{s}$ von der Fließgeschwindigkeit und der Einschnürung an der Schützöffnung bestimmt wird, kann diese als Beziehung $\mathrm{l}_{\mathrm{s}} / \mathrm{s}=\mathrm{f}\left(\mathrm{c}_{\mathrm{q}}\right)$ beschrieben werden. In Bild 5 sind die ausgewerteten Einflusslängen für verschiedene Verhältnisse von v/Vref dargestellt. Daraus ist zu erkennen, dass die Einfluss-
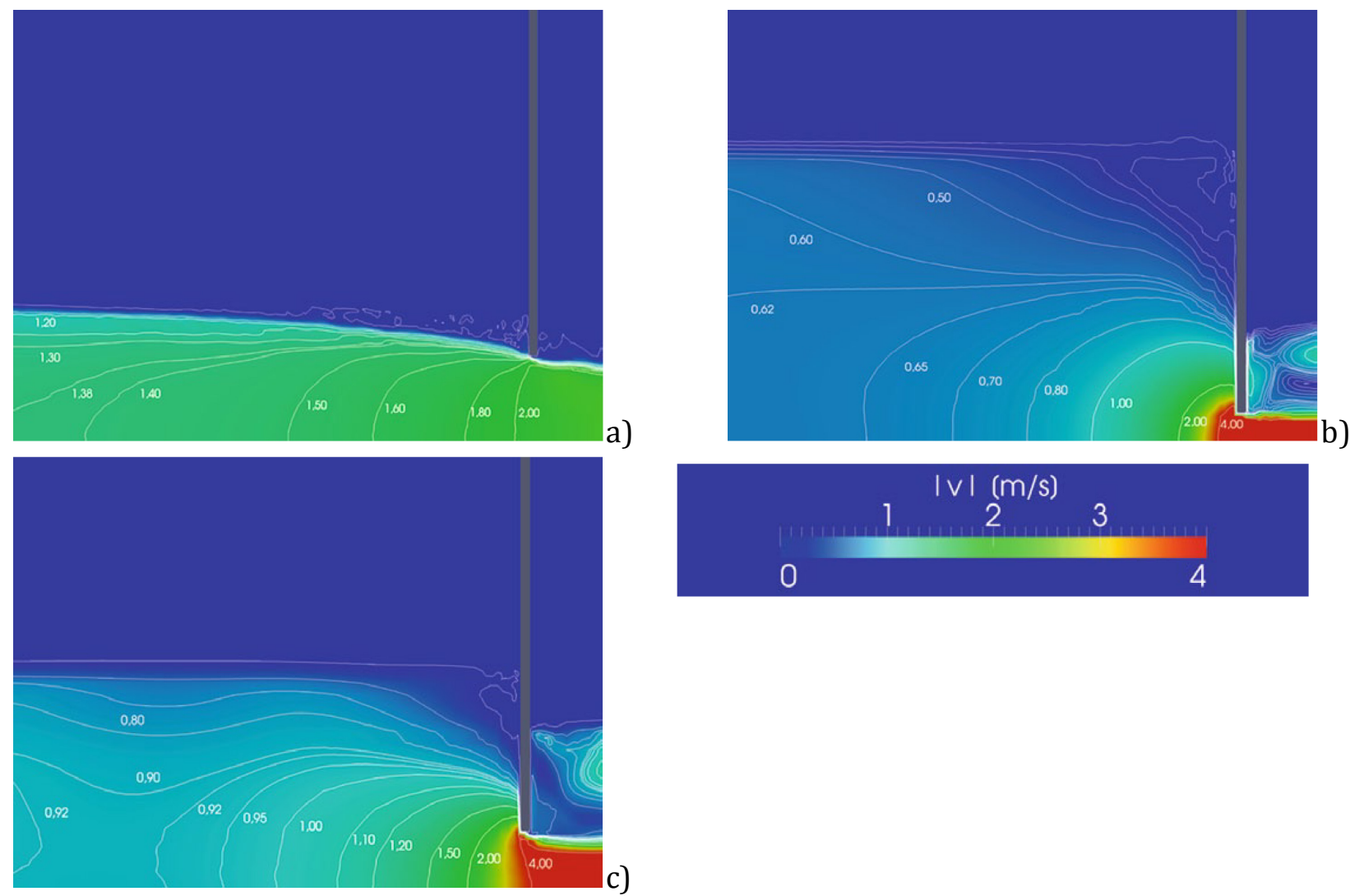

Bild 3: Isolinien der Fließgeschwindigkeit beim unterströmten Schütz für $Q=4,0 \mathrm{~m}^{3} / \mathrm{s}: \mathrm{a}$ ) s = $\left.0,70 \mathrm{~m}, \mathrm{c}_{\mathrm{q}}=0,49, \mathrm{~b}\right) \mathrm{s}=1,20 \mathrm{~m}, \mathrm{c}_{\mathrm{q}}=0,35$ und c) $\mathrm{s}=2,00 \mathrm{~m}, \mathrm{c}_{\mathrm{q}}=0,26$ 


\section{Autorenfassung}

Gebhardt, Gerstner, Thorenz: Der Einfluss über- und unterströmter Wehrverschlüsse auf den Sedimenttransport durch Stauhal tungen, 2014

Gebhardt, Gerster, Thorenz: Der Einfluss über- und unterströmter Wehrverschlüsse auf den Sedimenttransport durch Stauhaltungen. WasserWirtschaft 11 (2014), S. 16-22.

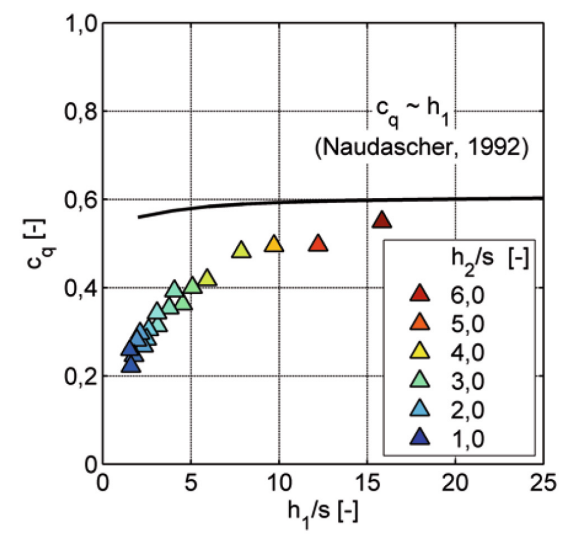

Bild 4: $\quad$ Berechnete Abflussbeiwerte $c_{q}$ der simulierten Zustände für das unterströmte Schütz im Vergleich zum theoretisch bzw. experimentell bestimmten Abflussbeiwert für ein Freispiegelschütz mit freiem Abfluss [6]

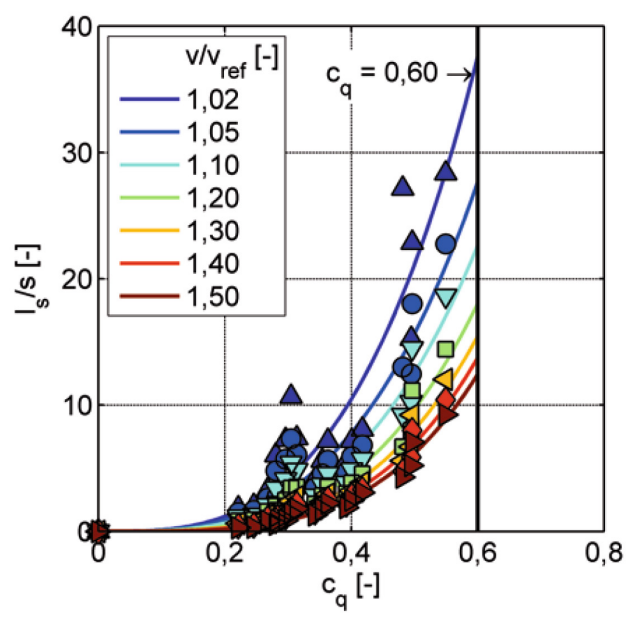

Bild 5: $\quad$ Einflusslänge $l_{s}$ beim unterströmten Schütz in Abhängigkeit vom Abflussbeiwert $c_{q}$ für verschiedene Abweichungen von $\mathrm{v} / \mathrm{v}_{\mathrm{ref}}$

länge mit steigendem Abflussbeiwert zunimmt: $F u ̈ r c_{q}=0,50$ liegt die sohlennahe Geschwindigkeit in einem Abstand von etwa $l_{s}=5 \mathrm{~s}$ etwa $50 \%$ über der Referenzgeschwindigkeit und bei ls = $20 \mathrm{~s}$ nur noch $2 \%$ darüber. Hierbei ist zu beachten, dass Abweichungen, die unter $10 \%$ der Referenzgeschwindigkeit liegen, schon stark durch numerische Einflüsse überlagert sind, wie die Streuungen in Bild 5 zeigen. Weiter oberstrom ist nahezu kein Einfluss mehr festzustellen. Bei einem kleineren 


\section{Autorenfassung}

Gebhardt, Gerstner, Thorenz: Der Einfluss über- und unterströmter Wehrverschlüsse auf den Sedimenttransport durch Stauhal tungen, 2014

Abflussbeiwert, beispielsweise durch Rückstau, verringert sich die Einflusslänge und damit auch die theoretische „Spülwirkung“ des geöffneten Verschlusses.

\section{2 Überströmtes Schütz}

In Bild 6 sind die Isolinien der Fließgeschwindigkeiten eines überströmten Schützes für drei exemplarischeZustände dargestellt. Bei konstantem Abfluss und nahezu konstanter Überfallhöhe nimmt die Verschlusshöhe w und damit $\mathrm{h}_{1}$ von a) nach c) zu. Da der Verschluss (numerisch) nicht belüftet ist, bildet sich zwischen Überfallstrahl und Schütz ein Unterdruck wodurch der Überfallstrahl angesaugt wird. Der Oberwasserstand $\mathrm{h}_{1}$ vergrößert sich mit zunehmender Verschlusshöhe, die sohlennahen Fließgeschwindigkeiten im Oberwasserverringern sich, wie beispielsweise an der Isolinie für v $=0,6 \mathrm{~m} / \mathrm{s}$ gut zu erkennen ist. Damit wird der Bereich potenzieller Anlandungen größer.

Für das überströmte Schütz wurden 27 Zustände mit Verschlusshöhen zwischen 2,0 und 6,0 m sowie den drei o. g. Abflüs-

Gebhardt, Gerster, Thorenz: Der Einfluss über- und unterströmter Wehrverschlüsse auf den Sedimenttransport durch Stauhaltungen. WasserWirtschaft 11 (2014), S. 16-22.
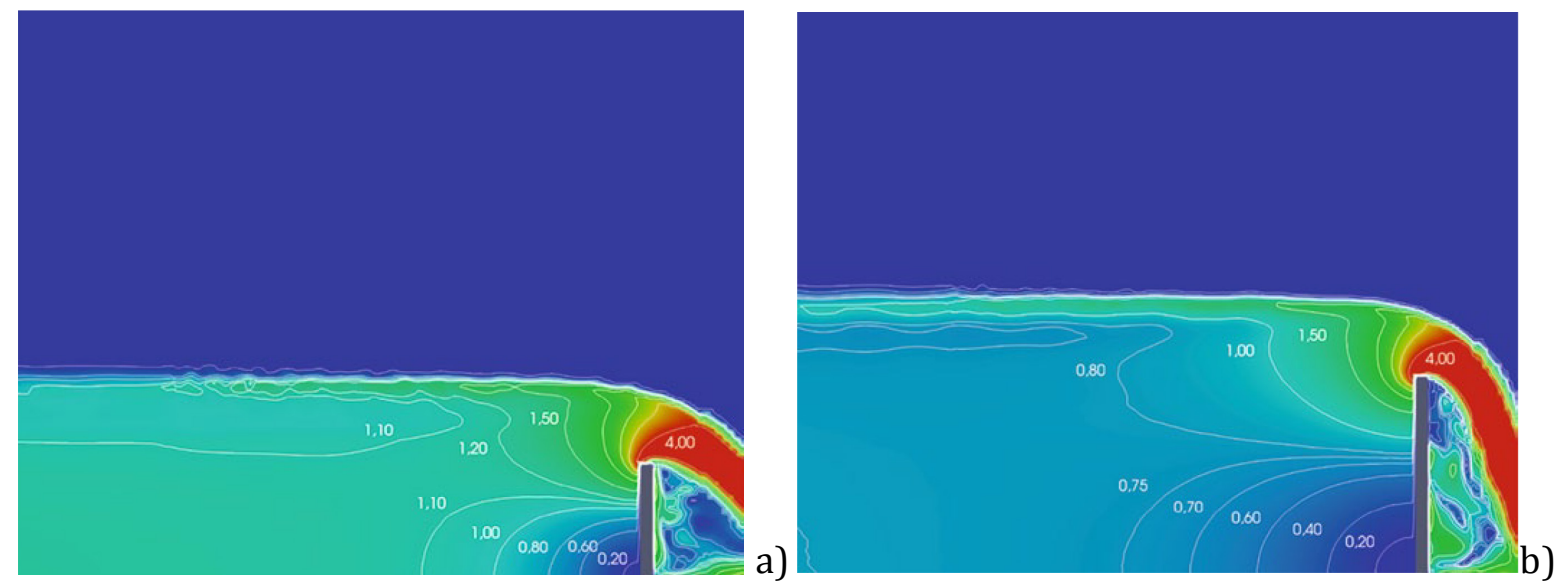


\section{Autorenfassung}

Gebhardt, Gerstner, Thorenz: Der Einfluss über- und unterströmter Wehrverschlüsse auf den Sedimenttransport durch Stauhal tungen, 2014
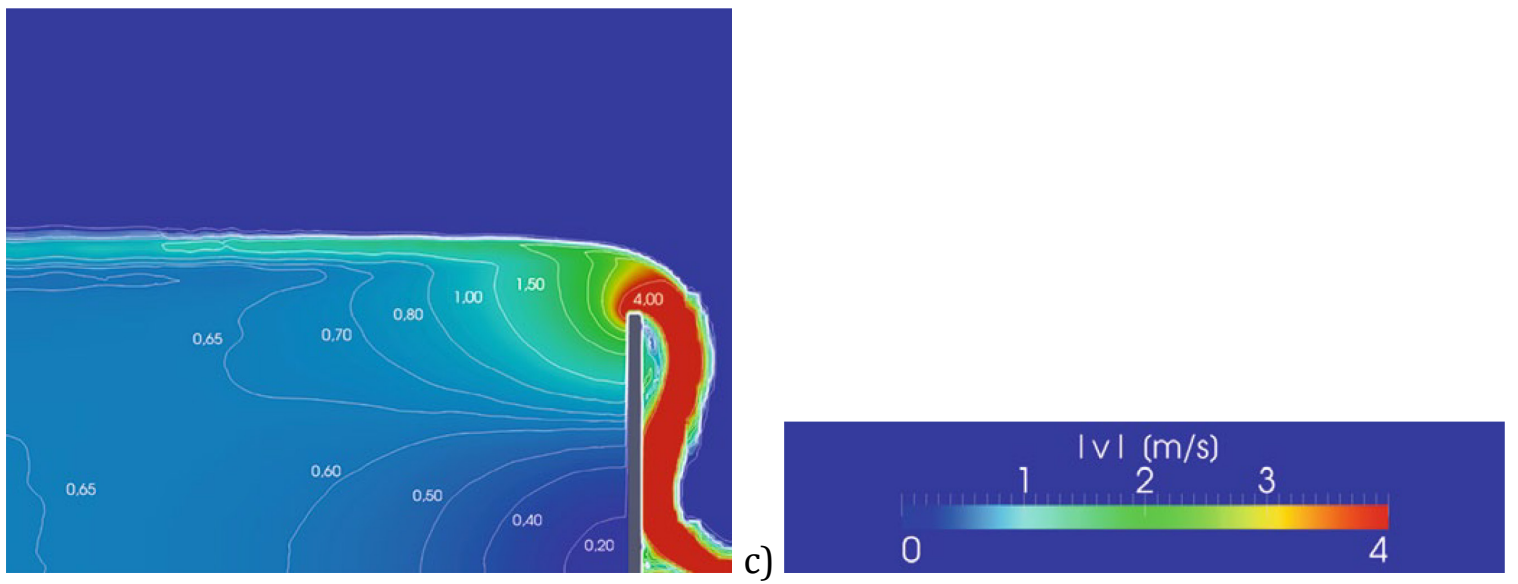

Bild 6: Isolinien der Fließgeschwindigkeit beim überströmten Schütz für $Q=4,0 \mathrm{~m}^{3} / \mathrm{s}$ :

a) $\left.\mathrm{w}=2,00 \mathrm{~m}, \mathrm{c}_{\mathrm{q}}=0,75, \mathrm{~b}\right) \mathrm{w}=3,50 \mathrm{~m}, \mathrm{c}_{\mathrm{q}}=0,83$ und c) $\mathrm{w}=4,60 \mathrm{~m}, \mathrm{c}_{\mathrm{q}}=0,88$

sen und Unterwasserständen betrachtet. Bild 7 zeigt die daraus berechneten Abflussbeiwerte $\mathrm{c}_{\mathrm{q}}$ in Abhängigkeit von der dimensionslosen Überfallhöhe $\left(\mathrm{h}_{1}-\mathrm{w}\right) / \mathrm{w}$ und dem dimensionslosen Unterwasserstand $h_{2} / w$ entsprechend folgender Gleichung:

$$
c_{q}=\frac{Q}{\frac{1}{3} \cdot b \cdot \sqrt{2 \cdot g}\left(h_{1}-w\right)^{\frac{3}{2}}}
$$

Es ist zu erkennen, dass $\mathrm{h}_{2}$ /w stets kleiner als „1“ ist, dadurch bei allen Zuständen vollkommener Überfall herrscht und $c_{\mathrm{q}}$ unabhängig von $\mathrm{h}_{2} / \mathrm{w}$ ist. Des Weiteren ist festzustellen, dass der Abflussbeiwert stets größer ist als der experimentell bestimmte Abflussbeiwert nach Rehbock [7]. Dies ist auf die fehlende Belüftung und den daraus resultierenden Unterdruck an der Strahlunterseite zurückzuführen, wodurch die Abflussleistung und damit $c_{q}$ vergrößert wird. Der Einfluss der fehlenden Belüftung nimmt mit zunehmendem $\left(\mathrm{h}_{1}-\mathrm{w}\right) / \mathrm{w}$ ab und die Abflussbeiwerte nähern nähern sich an die Ergebnisse der Formel von Rehbock [7] an.

In Bild 8 sind die ausgewerteten Einflusslängen für verschiedeneVerhältnisse von v/Vref dargestellt. Hierzu ist anzumerken, dass im Gegensatz zum unterströmten Schütz die sohlennahen Geschwindigkeiten in Fließrichtungabnehmen und das Verhältnis von $v / \mathrm{v}_{\mathrm{ref}}<1$ ist: Die Einflusslänge $\mathrm{l}_{\mathrm{w}}$ beschreibt hier also nicht einen Bereich erhöhter Fließgeschwindigkeiten, sondern einen Stillwasserbereich. Für $\mathrm{c}_{\mathrm{q}}=0,80$ liegt die sohlennahe Geschwindigkeit in einem Abstand von $\mathrm{l}_{\mathrm{w}}=\mathrm{w}$ etwa $50 \%$ unter der Referenzgeschwindigkeit und bei $l_{w}=8$ w nur noch $2 \%$ darunter. Hierzu ist anzumerken, dass die Einflusslängen bei belüftetem Überfallstrahlstrahl noch etwas kleiner wären.

\subsection{Diskussion der Ergebnisse}

Bei der Übertragung der Ergebnisse auf andere Wehranlagen ist zu berücksichtigen, dass Verschlussgeometrie und Unterwasserstand einen Einfluss auf den Abflussbeiwerthaben. Beim unterströmten Schütz liegt in den numerischen Untersuchungen immer ein Rückstaueinfluss vor. Das 


\section{Autorenfassung}

Gebhardt, Gerstner, Thorenz: Der Einfluss über- und unterströmter Wehrverschlüsse auf den Sedimenttransport durch Stauhal tungen, 2014

heißt, die Einflusslänge wäre ohne Rückstaueinfluss tendenziell größer. Ein überströmtes Schütz wird in der Praxis seitlich belüftet, so dass sich geringere Abflussbeiwerte ergeben als in den numerischen Untersuchungen. Die Einflusslänge wird dadurch tendenziell kleiner.

Es sei darauf hingewiesen, dass die in Bild 5 dargestellten Kurven bei $c_{q}=0,60$ enden, da dieser Abflussbeiwert nach Naudascher [6] einen Grenzwert für ein Freispiegelschütz mit senkrechter Stauwand und freiem Abfluss darstellt. Mit einer geneigten Stauwand können aber durchaus Abflussbeiwerte über 0,80 erreicht werden. Genauso bildet $c_{q}=0,577$ beim überströmten Schütz (Bild 7) einen theoretischen Grenzwert für kleine relativeWehrhöhen $w /\left(h_{1}-w\right)$, wenn dort die Grenzabflussverhältnisse auftreten.

Gebhardt, Gerster, Thorenz: Der Einfluss über- und unterströmter Wehrverschlüsse auf den Sedimenttransport durch Stauhaltungen.

WasserWirtschaft 11 (2014), S. 16-22.

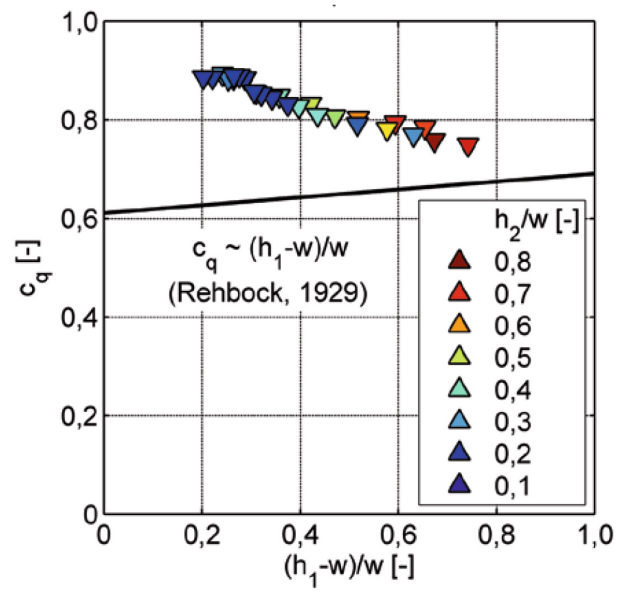

Bild 7: $\quad$ Berechnete Abflussbeiwerte $c_{q}$ der simulierten Zustände für das überströmte Schützim Vergleich zur experimentell bestimmten Formel für das scharfkantige Wehr mit vollkommenem Überfall [7] 


\section{Autorenfassung}

Gebhardt, Gerstner, Thorenz: Der Einfluss über- und unterströmter Wehrverschlüsse auf den Sedimenttransport durch Stauhal tungen, 2014

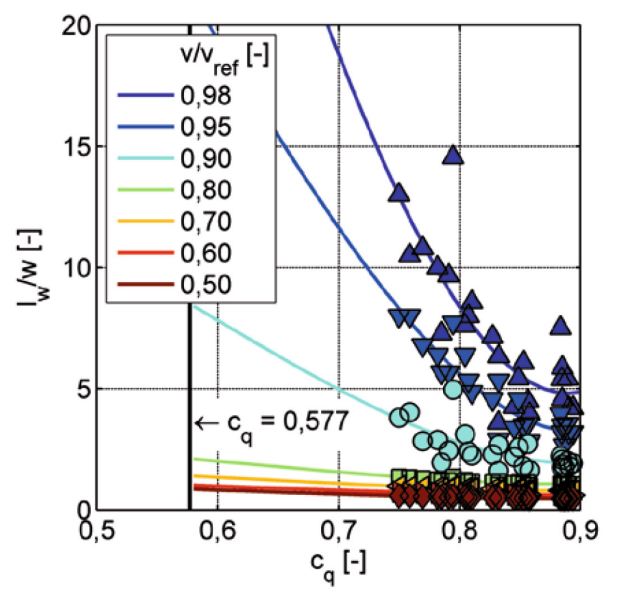

Bild 8: $\quad$ Einflusslänge $\mathrm{l}_{\mathrm{w}}$ beim überströmten Schütz in Abhängigkeitvom Abflussbeiwertc $\mathrm{c}_{\mathrm{q}}$ für verschiedene Abweichungen von $v / v_{\text {ref }}$

Beispielhaft sei ein Hubsenkschütz gegeben mit einerVerschlusshöhe bzw. einem Oberwasserstand von $\mathrm{h} 1=5,0 \mathrm{~m}$, einer Verschlussbreitevon 20,0 m, einer Öffnungsweitevon $\mathrm{s}=1,0 \mathrm{~m}$ und $\mathrm{c}_{\mathrm{q}}=0,50$. Aus Gl. (1) ergäbe sich folglich ein Abfluss von $\mathrm{Q}=99 \mathrm{~m}^{3} / \mathrm{s}$ und aus Bild 5 eine Einflusslänge von $\mathrm{l}_{\mathrm{s}} / \mathrm{s}=5\left(\mathrm{v} \mathrm{m} / \mathrm{V}_{\text {ref }}=1,50\right)$ bzw. $\mathrm{l}_{\mathrm{s}} / \mathrm{s}=20\left(\mathrm{v} / \mathrm{V}_{\text {ref }}=1,02\right)$ was $5,0 \mathrm{~m}$ bzw. 22,0 m entspricht. Geht man davon aus, dass ein Schütz etwa bei $\mathrm{s}=2 / 3 \mathrm{~h}_{1}$ aus dem Stau gefahren wird, weil es die Abflusskontrolle verliert, könnten in diesem Beispiel im Nahbereich der Wehranlage bis zu einer Einflusslänge von etwa 17,0 m (50\%) bzw. 67,0 m (2\%) sohlennah höhere Geschwindigkeiten erreicht werden.

Bei derselben Wehranlage, bei gleichen Randbedingungen und $c_{q}=0,80$ müsste das Schütz auf etwa $\mathrm{w}=3,4 \mathrm{~m}$ abgesenkt werden. Aus Bild 8 würde sich eine Einflusslänge von $\mathrm{l}_{\mathrm{w}} / \mathrm{w}=1\left(\mathrm{v} / \mathrm{v}_{\mathrm{ref}}=0,50\right)$ bzw. $\mathrm{l}_{\mathrm{w}} / \mathrm{w}=8\left(\mathrm{v} / \mathrm{V}_{\mathrm{ref}}=0,98\right)$ ergeben was Einflusslängen von 3,4 m bzw. 27,2 m entspricht. Das heißt, dass etwa $30 \mathrm{~m}$ oberhalb des überströmten Schützes praktisch kein Einfluss mehr feststellbar ist.

\section{Schlussfolgerungen}

An staugeregelten Gewässern ist das natürliche dynamische Transportgleichgewicht in den meisten Fällen gestört: Bei geringen bis mittleren Abflüssen findet Sedimentation statt, während bei Hochwasser abgelagerte Sedimente (teilweise) erodiert und ausgespült werden. Unterhal- 


\section{Autorenfassung}

Gebhardt, Gerstner, Thorenz: Der Einfluss über- und unterströmter Wehrverschlüsse auf den Sedimenttransport durch Stauhal tungen, 2014

Gebhardt, Gerster, Thorenz: Der Einfluss über- und unterströmter Wehrverschlüsse auf den Sedimenttransport durch Stauhaltungen. WasserWirtschaft 11 (2014), S. 16-22.

tungsbaggerungen sind erforderlich, um ausreichende Wassertiefen für die Schifffahrt und für den Hochwasserabfluss sicherzustellen, so dass sich in der Regel kein neuer Gleichgewichtszustand einstellen kann. Da sich neben den meisten Wehranlagen auch Laufwasserkraftwerke befinden, beschränkt sich der Einfluss des Wehrbetriebs bei einem normalen Ausbaugrad der Wasserkraftanlage auf den Mittel- bis Hochwasserbereich. Dabei wird die Ausbauwassermenge einer Wasserkraftanlage in Mitteleuropa im Schnittan etwa 30 bis 60 Tagen im Jahr erreicht bzw. überschritten $[8]$.

Die numerischen Untersuchungen zeigen, dass beispielsweisebei einem unterströmten Verschluss mit rückgestautem Abfluss $\left(c_{q}=0,50\right)$ die sohlennahen Geschwindigkeiten in einem Abstand, der etwa der 5-fachen Öffnungsweite entspricht, 50 \% über der Geschwindigkeit der ungestörten Anströmung liegen. Bei der 20-fachen Öffnungsweite liegen sie nur noch $2 \%$ darüber. Das heißt für die ingenieurpraktische Bedeutung, dass im Abstand von der 2- bis 3-fachen Wassertiefe im Oberwasser praktisch kein Einfluss durch den Verschluss mehr feststellbar ist.

Überträgt man die Ergebnisse auf typische Stauanlagen wird schnell klar, dass der Einflussbereich unabhängig vom Verschlusstyp im Verhältnis zur Stauhaltungslänge äußerst klein ist und nur Sedimente durch die Wehranlagetransportiertwerden, die auch bis in den Nahbereich der Wehranlage gelangen konnten. Die Wahl der Verschlüsse hat damit einen vernachlässigbaren Einfluss auf die (zeitweise) Unterbrechung des Sedimenttransports. Maßgeblich ist nicht die Gestaltung der Wehranlage, sondern die Stauregulierung an sich.

Es bleibt abschließend festzustellen, dass mit einem unterströmten Verschlusstyp im günstigsten Fall Ablagerungen im Nahbereich der Wehranlage remobilisiert werden können und ein Erosionstrichter entsteht. Untersuchungen zu Stauraumspülungen an Talsperren zeigen, dass die Wirkung einer Spülung umso besser ist, je länger sie erfolgt und je tiefer der Oberwasserstand abgesenkt wird [9]. Dies wird aber an den staugeregelten Wasserstraßen aufgrund ihrer Mehrfachnutzung und der möglichen ökologischen Folgen i. d. R. nicht zu realisieren sein. 


\section{Autorenfassung}

Gebhardt, Gerstner, Thorenz: Der Einfluss über- und unterströmter Wehrverschlüsse auf den Sedimenttransport durch Stauhal tungen, 2014

Michael Gebhardt, Nico Gerstner and Carsten Thorenz

The Impact of Overflow and Underflow Gates on the Sediment Transport in Impounded Rivers

Due to the EU Water Framework Directive which claims undisturbed sediment transport in addition to undisturbed migration of aquatic organisms, weir design and choice of gates is increasingly being asked in current projects of replacement constructions. The objective of the paper is to contribute generally to the understanding of sediment transport through impoundments and particularly to quantify the influence of overflow and underflow gates on the sediment transport.

Михаэль Гебхардт, Нико Герстнер и Карстен Торенц

Воздействие верховых и низовых затворов водосливной плотины на перемещение седиментов через подпорный бьеф

При актуальном планировании новых объектов, заменяющих старые, все чаще ставится вопрос о конструкции плотинного комплекса и выбора затворов, так как европейская Рамочная Директива по воде наряду с беспрепятственной миграцией живущих в воде организмов требует также ненарушенное перемещение седиментов через ступени подпора. Данная статья должна способствовать как пониманию вопросов перемещения седиментов через подпорный бьеф, так и определению и количественной оценке воздействия верховых и низовых затворов водосливной плотины на перемещение седиментов в частности.

\section{Literatur}

[1] DVWK (Hrsg): Verlandung von Flussstauhaltungen - Morphologie, Umweltaspekte und Fallbeispiele. Schriftenreihe des Deutschen Verbandes für Wasserwirtschaft und Kulturbau e. V. (1993), Nr. 105.

[2] Ferziger, P.; Peric, M.: Numerische Strömungsmechanik. Berlin: Springer, 2008.

[3] Gebhardt, M.; Pfrommer, U.; Belzner, F.; Eisenhauer, N.: 68 Jahre nach Jambor: Untersuchungen zum Einfluss einer Wehrschwelle. In: WasserWirtschaft 101 (2011), Heft 9, S. 14-19. 


\section{Autorenfassung}

Gebhardt, Gerstner, Thorenz: Der Einfluss über- und unterströmter Wehrverschlüsse auf den Sedimenttransport durch Stauhal tungen, 2014

[4] Jambor, F.: Mögliche Erhöhung der festen Wehrschwelle sowie Gestaltung der damit verbundenen Wehrkonstruktionen, im Besonderen des Sektorwehrs. In: Die Bautechnik (1959), Hefte 6 und 8, S. 221-228 u. 297-300.

[5] Kern, U.: Transport von Schweb- und Schadstoffen in staugeregelten Fließgewässern am Beispiel des Neckars. In: Mitteilungen des Instituts für Wasserbau der Universität Stuttgart (1997), Heft 93.

[6] Naudascher, E.: Hydraulik der Gerinne und Gerinnebauwerke. 2. A. Wien: Springer, 1992.

[7] Rehbock, T.: Wassermessung mit scharfkantigen Überfallwehren. In: Zeitschrift des Vereines Deutscher Ingenieure 73 (1929), Nr. 24, S. 817-826.

[8] Strobl, T.; Zunic, F.: Wasserbau. Berlin: Springer, 2006.

[9] Vischer, D.: Verlandung von Stauhaltungen und Speicherseen. In: Mitteilungen der Versuchsanstalt für Wasserbau, Hydrologie und Glaziologie der ETH Zürich (191), Nr. 53, S. 9-26.

[10] Westrich, B.: Fluvialer Feststofftransport - Auswirkungen auf die Morphologie und Bedeutung für die Gewässergüte. In: Schriftenreihe GWF Wasser, Abwasser (1998), Band 22. 